\title{
La implicación en la actividad física como fuente de felicidad en personas mayores
}

\author{
Alfonso J. García, M. Rocío Bohórquez y Macarena Lorenzo \\ Universidad de Sevilla (España)
}

\begin{abstract}
El principal objetivo de este estudio fue explorar la felicidad presente de personas mayores de 65 años, así como la relación que la implicación en la Actividad Física pudiera tener con ella. Los participantes cumplimentaron voluntariamente un cuestionario de datos sociodemográficos, La Escala de Actividad Física de la Batería STEPS 2.1 (OMS, 2012) y la Escala de Felicidad (Godoy-Izquierdo y Godoy, 2006). Los resultados muestran que la felicidad actual de los mayores es inferior a la felicidad pasada. Además, se demuestra que la práctica actual de actividad física incide en la felicidad de los mayores, siendo más felices aquellos más activos en esta etapa de su vida. Finalmente, haber estado implicado en la práctica de actividad física en algún momento del ciclo vital (en el pasado, en el presente o de manera continua) está relacionado con un nivel mayor de felicidad que haber tenido un estilo de vida sedentario, los mecanismos gracias a los cuales la práctica de actividad física tiene este papel protector de la felicidad están por determinar. Este papel protector de la Actividad Física ha de ser tenido en cuenta en el diseño de los programas de promoción e intervención en el envejecimiento activo y positivo de la población.
\end{abstract}

Palabras clave: Felicidad, bienestar, ejercicio físico, vejez.

Happiness and physical activity in the elderly. The overall objective of this study was to explore past and present happiness from people over 65 years old, and the relationship that physical activity may have with it. Participants voluntarily completed a sociodemographic questionnaire, the Physical Activity Scale Battery STEPS 2.1 (WHO, 2012) and the Scale of Happiness (Godoy and Godoy-Izquierdo, 2006). The results show that present happiness is lower than last. Furthermore, it is shown that the current practice of physical activity affects the happiness of the greatest, being happiest those most active at this stage of his life. Finally, having been involved in physical activity at some point in the life cycle (past, present or continuously) is related to a higher level of happiness than that related to a sedentary lifestyle; the mechanisms involved in this protective role of happiness must be determined. This protective role of physical activity has to be taken into account in the design of programs and intervention promoting active and positive aging population.

Keywords: Happiness, well-being, exercise, old age.

Correspondencia: Alfonso J García. Dpto. Psicología Social, Facultad de Psicología. Universidad de Sevilla. C/ Camilo José Cela, s/n. C.P.: 41008 Sevilla (España). E-mail: alfonsoj@ us.es 
Calidad de vida, bienestar subjetivo y felicidad son términos que, aunque a menudo han sido utilizados indistintamente, denotan significados diferentes (Veenhomen, 2000). Atendiendo al modelo de bienestar subjetivo de Diener (1984, Diener y Suh, 1998), el bienestar subjetivo incluiría respuestas emocionales -balance afectivo- y juicios globales de la satisfacción con la vida, ambos en relación a dominios concretos (familia, trabajo, etcétera). Desde esta óptica, la felicidad sería un resultado en la evaluación del bienestar subjetivo caracterizado por un balance afectivo positivo y un juicio global de satisfacción con la vida en el dominio del yo (Diener y Suh 1998).

Dos tipos de factores han sido evaluados en su relación con el bienestar subjetivo: de un lado variables sociodemográficas y de otro, factores psicosociales. En lo relativo a las variables sociodemográficas se ha abordado la influencia de la edad (Pinquart, 2001; McMahan y Estes, 2012), el género (Pinquart y Sorensen, 2001), la raza (Liang, Lawrence y Bollen, 1987; Mckenzie y Campbell, 1987), el estatus marital (Haring-Hidore, Stock, Okun y Witter, 1985; Waldinger y Schultz, 2010), las relaciones familiares (Diener y Suh, 1998; Pinquard y Sorensen, 2000), las relaciones sociales (Pinquart y Sorensen, 2000), la situación laboral (VanPraag, Frijters y Ferrer-iCarbonell, 2003) o el estado de salud (Okun, Stock, Haring y Witter, 1984; VanPraag et al., 2003). A pesar de este amplio rango de factores estudiados, se ha postulado una escasa influencia de las variables sociodemográficas en la percepción del bienestar subjetivo (Diener y Suh, 1998; Diener, Suh, Lucas y Smith, 1999).

Por contra, los factores psicosociales han sido identificados como la mayor fuente de variabilidad en relación al bienestar subjetivo (Godoy-Izquierdo, Lara, Vázquez, Araque y Godoy, 2012). Las variables psicosociales relacionadas con el bienestar subjetivo que más impacto han tenido en la literatura han sido el optimismo (Ferreira y Sherman, 2007), las estrategias de afrontamiento (Caprara y Steca, 2005), la autoestima (García, Marín y Bohórquez, 2012), la autoeficacia (Caprara y Steca, 2005; Piquart y Sorensen, 2000) y el control percibido (Diener et al., 1999).

El bienestar subjetivo parece ser bastante estable a lo largo de la vida (Diener et al., 1999; Suh, Diener y Fujita, 1996; Suh, Diener, Oishi y Triandis, 1998), aunque se ha señalado la existencia de un patrón evolutivo en forma de U invertida (GodoyIzquierdo et al., 2012; Godoy-Izquierdo, Martínez y Godoy, 2009), de modo que a partir de los 40 años aproximadamente el bienestar subjetivo parece ser menor para mejorar alrededor de los 50 años y alcanzar posteriormente niveles semejantes a los de la mitad de la adultez (Blanchflower y Oswald, 2008; Godoy-Izquierdo et al., 2012).

En lo relativo a la vejez propiamente dicha, la visión tradicional ha asociado esta etapa de la vida con infelicidad y ausencia de bienestar subjetivo (Lang y Heckhausen, 2001; Lelkes, 2008; Mrockzek y Spiro, 2005), aunque en los últimos años diversos autores han considerado estos resultados inconclusos y no determinantes 
(Cartesen, Pasupathi, Mayr y Nesselroade, 2000; Ehrlich e Isaacowtiz, 2002; García, 2011).

Godoy-Izquierdo et al. (2012) indican que los principales predictores de bienestar subjetivo en la adultez tardía son la satisfacción con el estado de salud, el estatus marital, las relaciones sociales, la satisfacción de las necesidades básicas, las habilidades personales, la autoeficacia y el ocio activo. Partiendo de la importancia éstas variables -especialmente estado de salud y ocio activo-, la práctica de actividad física se postula como un elemento a tener en cuenta en la evaluación e intervención en bienestar en los mayores (Guillén, 1996; Menec, 2003; Pawlowsky, Downward y Rasciute, 2011).

La práctica de actividad física por parte de los mayores de 65 años ha sido relacionada una mayor calidad de vida (Rejeski, Brawley y Shumaker, 1996; Sánchez, Ureña y Garcés de los Fayos, 2002) llegando incluso a postularse que esta mejoría seguiría un patrón lineal, de modo que la mayor calidad de vida correspondería a sujetos con elevados niveles de práctica de actividad física (Jürgens, 2006). Para Elavsky et al. (2005), esta relación tendría no sólo efectos a corto plazo sino también a largo plazo extendiéndose durante un período temporal de un año. Por el contrario, el sedentarismo en personas mayores se ha relacionado con una disminución de sus capacidades físicas, cognitivas y psicológicas (Guillén, 1996; Guillén y Sánchez, 2010).

La relación entre actividad física y calidad de vida está mediada, según diferentes autores (Márquez, Hu, Jerome y Diener, 2005; McAuley et al., 2006), por los beneficios consecuentes de la implicación activa en el ejercicio, del mismo modo es posible entender que la relación entre sedentarismo y disminución de la calidad de vida se encontraría mediada por los mismos mecanismos. Beneficios de la actividad física como la autoeficacia (Dionigi, 2007; McAuley, Blissmer, Katula, Duncan y Mihalko, 2000), la autoestima (García et al., 2012), el afecto positivo (Salmon, Owen, Crawford, Bauman y Sallis, 2005) o la satisfacción con la vida (Meléndez, Tomás, Oliver y Navarro, 2008) promocionarían el bienestar subjetivo o lo disminuirían ante su inexistencia/desaparición.

Mientras que la relación entre calidad de vida y/o bienestar subjetivo y la práctica de actividad física ha sido previamente abordada en la literatura, no ha sido posible encontrar estudios que relacionen felicidad y ejercicio. Así, el objetivo de este estudio fue explorar la felicidad -presente y pasada- de las personas mayores de 65 años así como la relación que la práctica de actividad física pudiera tener con ella. De este objetivo general se desprendieron las siguientes hipótesis:

Los mayores que realizan actividad física estimarán mayor su felicidad que aquellos sedentarios.

Los mayores estimarán mayor su felicidad cuanta más actividad física realicen. 
Aquellos que más actividad física realicen valorarán en mayor medida la contribución de (I) la salud, bienestar y calidad de vida, (II) los hábitos y estilo de vida y (III) la autonomía e independencia personal en su felicidad.

\section{MÉTODO}

\section{Participantes}

La muestra -36 hombres y 68 mujeres- se formó por accesibilidad, contactando con personas mayores en diferentes espacios públicos. La edad media de los participantes fue 76.34 años (Mín.=64, Máx.=91, DT=8.10).

En cuanto al historial de actividad física de los participantes 26 de ellos (25\%) siempre han estado involucrados en algún tipo de actividad física o deportiva, 8 de ellos (7.7\%) únicamente lo hicieron en el pasado, 44 realizan en esta etapa de su vida actividad física o deportiva por primera vez (42.3\%) mientras que 26 participantes $(25 \%)$ jamás se han involucrado en actividad física ya sea de manera formal o informal.

\section{Instrumentos}

Se desarrolló ex profeso una ficha de datos sociodemográficos que incluyó edad, género, estado civil, número de hijos, tipo de residencia, personas con las que se convive en el lugar de residencia, situación laboral, nivel académico e historial de actividad física y deporte.

Para la evaluación de los hábitos de actividad física/sedentarismo se empleó el Global Physical Activity Questionnaire -GPAQ- 2.0 en versión española (OMS, 2012). El GPAQ se compone de 16 ítems de los cuales 6 son de obligado cumplimiento mientras que los 10 restantes se cumplimentan en caso de respuestas afirmativas a preguntas llave. Este instrumento tiene en cuenta la actividad física realizada, su intensidad y el contexto en el que ésta se realiza (trabajo, desplazamiento u ocio), además, incluye una estimación del tiempo empleado en comportamientos sedentarios.

La evaluación de la felicidad y las fuentes de ésta se realizó mediante la Escala de Felicidad (Godoy-Izquierdo, Lara, Vázquez, Araque y Godoy, 2006). Esta escala se compone de 22 ítems que el sujeto ha de valorar en una rango 0-10. De estos ítems, 2 evalúan la felicidad actual y pasada y los 20 restantes la influencia de diferentes variables (como familia y relaciones familiares, hábitos y estilos de vida, herencia genética, etc.) en la felicidad actual de la persona.

\section{Procedimiento}

Se contactó con los mayores entrevistados en residencias de mayores, instalaciones deportivas municipales y diferentes zonas públicas como mercados, parques, calles céntricas transitadas, etc. Una vez realizada la presentación del 
entrevistador, se procedía a informar a los participantes de los objetivos del estudio, el formato de participación en el que se les deseaba involucrar y el tratamiento confidencial de sus datos. Tan sólo se entrevistó a aquellas personas que dieron su consentimiento y mostraron signos de adecuados estados de alerta y comprensión. Los cuestionarios se cumplimentaron en todas las ocasiones por un entrevistador entrenado que leía cada ítem al participante y procedía, cuando era necesario, a explicar las dudas que cada uno de ellos pudiera suscitar.

\section{Análisis de datos}

Los datos arrojados por las entrevistas fueron codificados y volcados en una matriz de SPSS 20.0 (IBM Corporation, 2012). De forma previa al análisis de datos se realizaron pruebas de normalidad y homocedasticidad de la muestra con el fin de tomar decisiones acerca de las pruebas estadísticas a emplear. Se determinó una distribución no normal de la muestra, así como la no homogeneidad de varianzas para las variables principales del estudio (pruebas de Kolmogorov-Smirnov y Levene, $p<.05$ ). Por ello se decidió utilizar pruebas no paramétricas en los análisis estadísticos realizados para este estudio. La primera hipótesis fue analizada mediante una prueba Wilcoxon de comparación de grupos dependientes, mientras que las hipótesis segunda y tercera se examinaron con pruebas Kruskall-Wallis para comparación de grupos independientes, para completar los análisis de la tercera hipótesis se efectuaron contrastes a posteriori Mann-Whitney.

\section{RESULTADOS}

La Tabla 1 muestra los principales descriptivos de las variables objeto de estudios:

Tabla 1. Descriptivos de las principales variables objeto de estudio

\begin{tabular}{lrrrrr}
\hline & $N$ & Mín. & Máx. & $M$ & $D T$ \\
\hline Feliz_actualidad & 104 & .00 & 10.00 & 6.23 & 2.86 \\
\hline Total Actividad Física diaria* & 104 & 1.00 & 4,00 & 2.44 & 1.30 \\
\hline Influencia de la salud, el bienestar y la calidad de vida. & 104 & 2.00 & 10.00 & 8.19 & 2.30 \\
\hline Influencia de los hábitos y el estilo de vida. & 104 & 1.00 & 4.00 & 2.50 & 1.03 \\
\hline Influencia de la autonomía e independencia personal. & 104 & 0 & 10.00 & 8.29 & 2.23 \\
\hline *1=menos de 60', 2=entre 60' y 90', 3=entre 90'y 120', 4=más de 120'. & & &
\end{tabular}

Para indagar en la influencia de la actividad física realizada por los mayores de 65 años en el estado actual de felicidad se ejecutaron pruebas no paramétricas de comparación de grupos independientes. Los resultados, tabla 2, indicaron diferencias significativas en la felicidad actual de los participantes en función de su implicación en tareas de ejercicio físico, estimando mayor su felicidad aquellos que realizan actualmente algún tipo de actividad física. 
Tabla 2. Descriptivos e inferenciales para felicidad actual en función de la realización/no de actividad física

\begin{tabular}{lcccccc}
\hline & \multicolumn{2}{c}{ Sedentarismo } & \multicolumn{2}{c}{ AF } & \multirow{2}{*}{$X^{2}$} & \multirow{2}{*}{$p$} \\
\cline { 2 - 6 } & $M$ & $D T$ & $M$ & $D T$ & & \\
\hline Felicidad actual & 4.65 & 2.53 & 7 & 2.70 & 22.63 & $.00^{* *}$ \\
\hline ** $<$ p 01 & & & & & &
\end{tabular}

Con el objetivo de establecer la posible relación entre cantidad de Actividad Física realizada por los mayores participantes y su felicidad actual, se realizaron pruebas no para métricas de comparación de grupos independientes. Los resultados mostraron diferencias significativas $\left(X^{2}=20.90, p=.00\right)$ en la felicidad de los mayores encuestados en función de la cantidad de actividad física realizada diariamente. Como se observa en la tabla 3 , aquellos que realizan menos de sesenta minutos de actividad física se consideran menos felices que aquellos que se ejercitan, en general, más de noventa minutos al día, en la misma línea, aquellos que se ejercitan entre sesenta y noventa minutos se consideran menos felices que aquellos que lo hacen durante más de ciento veinte minutos.

Tabla 3. Descriptivos e inferenciales para la felicidad actual en función de la cantidad de actividad física realizada diariamente

\begin{tabular}{|c|c|c|c|c|c|c|c|c|c|c|}
\hline \multicolumn{2}{|c|}{ Menos de 60 (1) } & \multicolumn{2}{|c|}{$60^{\prime}-90^{\prime}(2)$} & \multicolumn{2}{|c|}{$90^{\prime}-120^{\prime}(3)$} & \multicolumn{2}{|c|}{ Más de $120^{\prime}(4)$} & \multirow{2}{*}{ Comparaciones post hoc } & \multirow{2}{*}{$U$} & \multirow{2}{*}{$p$} \\
\hline$M$ & $D T$ & $M$ & $D T$ & $M$ & $D T$ & $M$ & $D T$ & & & \\
\hline \multirow{6}{*}{4.80} & \multirow{6}{*}{2.86} & \multirow{6}{*}{6.50} & \multirow{6}{*}{1.31} & \multirow{6}{*}{6.78} & \multirow{6}{*}{2.90} & \multirow{6}{*}{7.53} & \multirow{6}{*}{2.56} & $1-2$ & 152 & .05 \\
\hline & & & & & & & & $1-3$ & 202 & $.00^{* *}$ \\
\hline & & & & & & & & $1-4$ & 298 & $.00^{* *}$ \\
\hline & & & & & & & & $2-3$ & 82 & .26 \\
\hline & & & & & & & & $2-4$ & 122 & $.04 *$ \\
\hline & & & & & & & & $3-4$ & 256 & .33 \\
\hline
\end{tabular}

$* * p<.01, * p<.05$

Para indagar en la influencia de la actividad física realizada por los mayores de 65 años en el (I) la salud, el bienestar y la calidad de vida, (II) los hábitos y estilos de vida y (III) la autonomía e independencia personal como fuentes de felicidad, se ejecutaron pruebas no paramétricas de comparación de grupos independientes. Los resultados indicaron la existencia de diferencias significativas en la contribución estimada en la felicidad actual de -Factor de Felicidad I- la salud, el bienestar y la calidad de vida $\left(X^{2}=16.42, p=.00\right)$, -Factor de Felicidad II- los hábitos y estilos de vida $\left(X^{2}=22.64, p=.00\right)$ y - Factor de Felicidad III- la autonomía e independencia personal $\left(X^{2}=12.57, p=.01\right)$. Las tablas 4 y 5 muestran respectivamente resultados descriptivos e inferenciales que señalan que:

Respecto a la percepción de salud, bienestar y la calidad de vida. Los mayores que se involucran en actividad física durante menos de sesenta minutos al día consideran que este factor influye en su felicidad en menor medida que aquellos que se ejercitan más de noventa minutos diarios. 
En lo relativo a la importancia de los hábitos y estilos de vida como factor de felicidad. Los mayores que se involucran en actividad física durante menos de sesenta minutos al día consideran que este factor influye en su felicidad en menor medida que aquellos que se ejercitan más de noventa minutos diarios, además, aquellos mayores que hacen ejercicio durante más de 120 minutos consideran más importante este factor en su felicidad que aquellos que no lo hacen en intervalos de entre sesenta y noventa minutos.

Para la importancia de la autonomía y la independencia personal en la felicidad, aquellos que practican más de ciento veinte minutos diarios consideran que éste factor aporta mayor felicidad que aquellos mayores que se implican en actividad física durante menos de sesenta minutos o entre noventa y ciento veinte minutos.

Tabla 4. Descriptivos para la contribución de diferentes factores de personalidad en función de la cantidad de actividad física realizada diariamente

\begin{tabular}{lcccccc}
\hline & \multicolumn{2}{c}{ Salud, bienestar y calidad de vida. } & Hábitos y estilos de vida. & \multicolumn{2}{c}{ Autonomía e independencia personal. } \\
\cline { 2 - 6 } & $M$ & $D T$ & $M$ & $D T$ & $M$ & $D T$ \\
\hline Menos de 60' (1) & 7.50 & .42 & 6.90 & 2.02 & 7.90 & 8.67 \\
\hline $60^{\prime}-90^{\prime}(2)$ & 6.50 & 2.75 & 7.33 & 2.15 & 7.11 \\
\hline $90^{\prime}-120^{\prime}(3)$ & 9.22 & 1.52 & 7.89 & 2.40 & 1.15 \\
\hline Más de 120'(4) & 9.06 & 1.22 & 8.82 & 1.27 & 9.24 \\
\hline
\end{tabular}

Tabla 5. Inferenciales para la contribución de diferentes factores de personalidad en función de la cantidad de actividad física realizada diariamente

\begin{tabular}{|c|c|c|c|c|c|c|}
\hline & \multicolumn{2}{|c|}{$\begin{array}{l}\text { Salud, bienestar y } \\
\text { calidad de vida }\end{array}$} & \multicolumn{2}{|c|}{$\begin{array}{l}\text { Hábitos y estilos de } \\
\text { vida }\end{array}$} & \multicolumn{2}{|c|}{$\begin{array}{c}\text { Autonomía e } \\
\text { independencia personal }\end{array}$} \\
\hline & $U$ & $p$ & $U$ & $p$ & $U$ & $p$ \\
\hline Menos de 60'-entre 60'y 90' & 182 & .20 & 234 & .89 & 212 & .52 \\
\hline Menos de 60'-entre 90'y $120^{\prime}$ & 220 & $.01 *$ & 210 & $.01 *$ & 284 & .25 \\
\hline Menos de $60^{\prime}$-más de $120^{\prime}$ & 474 & $.02 *$ & 256 & $.00 * *$ & 476 & $.02 *$ \\
\hline Entre $60^{\prime}$ y $90^{\prime}$-entre $90^{\prime}$ y $120^{\prime}$ & 38 & $.00 * *$ & 88 & .38 & 68 & .08 \\
\hline Entre $60^{\prime}$ y $90^{\prime}$-más de $120^{\prime}$ & 84 & $.01 *$ & 116 & $.02 *$ & 146 & .11 \\
\hline Entre $90^{\prime}$ y $120^{\prime}$-más de $120^{\prime}$ & 254 & .25 & 238 & .17 & 138 & $.00 * *$ \\
\hline
\end{tabular}

\section{DISCUSIÓN}

Las personas felices viven más y son más autónomas que las personas infelices (Danner, Snowdon y Friesen, 2001; Lucas, 2007), aunque esta relación está claramente mediada por la morbilidad prevalente y las conductas de salud como la práctica de actividad física (Castillo, Ortega y Ruiz, 2005; Godoy-Izquierdo et al., 2012). Así, el objetivo principal de este estudio fue explorar la felicidad presente de las personas mayores de 65 años así como la relación que la práctica de actividad física pudiera tener con ella.

La primera hipótesis postulaba que los mayores que realizan actividad física estimarían mayor su felicidad que los sedentarios, relación que se ve confirmada por los datos al estimar su felicidad en algo más de 2 puntos de media (en una escala 0-10) para los mayores de 65 años que practican algún tipo de actividad física frente a aquellos que 
tienen un estilo de vida sedentario. Estos resultados confirman los encontrados por Rejeski et al. (1996), Sánchez et al. (2002), Guillén (1996) o Guillén y Sánchez (2010). Desde nuestro punto de vista, la relación felicidad-ejercicio es expresión de los beneficios de la actividad física sobre el bienestar físico, psicológico y social de la población general y de la población mayor en particular como ya indicaran, entre otros, Campos y Huertas (2003) o Capdevila (2005), en este sentido, la mejora de la condición física, las habilidades de autonomía, la auto-eficacia y las estrategias de afrontamiento podrían ser algunos de los productos de la actividad física más beneficiosos para un envejecimiento con bienestar subjetivo, aunque este aspecto debe ser comprobado en futuras investigaciones.

La segunda hipótesis proponía que a mayor cantidad de Actividad Física, mayor sería la felicidad actual estimada por los mayores. Los resultados obtenidos permiten confirmar esta hipótesis, mostrando diferencias significativas entre diferentes implicaciones temporales en tareas de actividad física indistintamente de su origen (por trabajo, desplazamiento o actividades de ocio). Estos resultados son coherentes con los hallados por Jürgens (2006), Rejeski, Brawley y Shumaker (1996) o Sánchez, Ureña y Garcés de los Fayos (2002) en relación a la calidad de vida, si atendemos a la felicidad como un producto de la evaluación de la calidad de vida (Diener, 1984) es lógico deducir que ambas se encontrarían correlacionadas como insinúan los resultados hallados.

En cuanto a la tercera hipótesis, postulaba que aquellos que más actividad física realicen valorarán en mayor medida la contribución de (I) la salud, bienestar y calidad de vida, (II) los hábitos y estilo de vida y (III) la autonomía e independencia personal en su felicidad. Esta hipótesis es confirmada por los resultados en la línea de lo afirmado por Godoy et al. (2012), quienes destacaban esos factores como fuentes de felicidad en personas mayores, en este caso, además, es posible afirmar que de forma general cuanto mayor sea la implicación temporal diaria de los participantes en actividad física, mayor será la contribución percibida de estos factores a la felicidad.

Entre las limitaciones de este estudio cabe destacar la limitación del tamaño de la muestra, que no sólo resta representatividad a la misma sino que impide el uso de pruebas paramétricas de decisión estadística, en base a las cuales es posible explorar las relaciones planteadas con mayor precisión. Además, es necesario señalar la dificultad de la cumplimentación de las pruebas por parte de los participantes que, aunque asistidos por los investigadores, mostraron dificultades en la comprensión de algunos conceptos, en la limitación de su respuesta a las preguntas y en la utilización de escalas cuantitativas para la evaluación de conceptos cualitativos.

Futuras investigaciones deberían partir necesariamente de una adaptación de los instrumentos a la población mayor de 65 años y de una muestra de mayor tamaño. Además, repicar este estudio agrupando a los participantes en función de su voluntariedad/obligatoriedad en la práctica de actividad física permitiría clarificar el 
sentido de ocio o tratamiento que este ejercicio tuviera para los mayores y quizás correlacionarlo con la felicidad - presente y pasada- estimada. Por otro lado, una metodología longitudinal permitiría hacer un seguimiento estricto de las conductas de actividad física de los participantes y de su felicidad percibida, perspectiva que facilitaría confirmar el papel protector de la práctica de ejercicio en la felicidad de los mayores y, quizás, entender los mecanismos que median en dicha relación.

A pesar de las limitaciones señaladas y de las diferentes investigaciones que aún quedan por desarrollar, nuestros resultados permiten suponer un papel protector de la actividad física en el bienestar subjetivo de las personas mayores y por lo tanto de su felicidad. Este papel protector es fundamental en el diseño de los programas de promoción e intervención en el envejecimiento activo y positivo de la población.

\section{REFERENCIAS}

Blanchflower, D.G. y Oswald, A.J. (2008). Is well-being U-shaped over the life cycle? Social Science and Medicine, 66, 1733-1749.

Campos, J. y Huertas, F. (2003). Efectos de un programa de ejercicio físico sobre el bienestar psicológico de mujeres mayores de 55 años. Revista de Psicología del Deporte, 12(1), 7 26.

Capdevila, L. (2005). Actividad Física y Estilo de Vida Saludable. Girona: Documenta Universitaria.

Caprara, G.V. y Steca, P. (2005). Affective and social self-regulatory efficacy beliefs as determinants of positive thinking and happiness. European Psychologist, 10, 275-286.

Carstensen, L., Pasupathi, M., Mayr, U. y Nesselroade, J.R. (2000). Emotional experience in everyday life across the adult life span. Journal of Personality and Social Psychology, 79, 644-654.

Castillo, M.J., Ortega, F.B. y Ruiz, J. (2005). Mejora de la forma física como terapia antienvejecimiento. Medicina Clínica (Barc),124(4), 146-155.

Danner, D.D., Snowdon, D.A. y Friesen, W.V. (2001). Positive emotions in early life and longevity: Findings from the nun study. Journal of Personality and Social Psychology, 80, 804-813.

Diener, E. (1984). Subjective well-being. Psychological Bulletin, 95(3), 542-575.

Diener, E. y Suh, E.M. (1998). Subjective well-being and age: An international analysis. En K. Schaie, K. Warner y L.M. Powell (Eds.), Annual Review of Gerontology and Geriatrics: Focus on Emotion and Adult Development, 17, 304-324.

Diener, E., Suh, E.M., Lucas, R.E. y Smith, H.L. (1999). Subjective well-being: three decades of progress. Psychological Bulletin, 125(2), 276-302.

Dionigi, R. (2007). Resistance training and older adults' beliefs about psychological benefits: the importance of self-efficacy and social interaction. Journal of Sport and Exercise Psychology, 29(6), 723-746.

Ehrlich, B.S. e Isaacowitz, D.M. (2002). Does subjective well-being increase with age? Perspectives in Psychology, 5, 20-26.

Elavsky, S., McAuley, E., Motl, R.W., Konopack, J.F., Márquez, D.X., Hu, L., Jerome, G.J. y Diener, E. (2005). Physical Activity Enhances Long-Term Quality of Life in Older Adults: Efficacy, Esteem, and Affective Influences. Annals of Behavioral Medicine, 30(2), 138-145. 
Fernández-Ballesteros, R. (2004). La psicología de la vejez. Encuentros Multidisciplinares, 6(16), 11-22.

Fernández-Ballesteros, R., Zamarrón, M.D. y Ruiz, M.A. (2001). The contribution of sociodemographic and psychosocial factors to life satisfaction. Aging and Society, 21, 25-43.

Ferreira, V.M. y Sherman, A.M. (2007). The relationship of optimism, pain and social support to wellbeing in older adults with osteoarthritis. Aging \& Mental Health, 11, 89-98.

García, A.J. (2011). Variables Psicosociales que inciden en la calidad de vida del alumnado participante en programas universitarios de mayores (Tesis Doctoral).Universidad de Sevilla: Sevilla.

García, A.J., Marín, M. y Bohórquez, M.R. (2012). Autoestima como variables psicosocial predictora de actividad física en personas mayores. Revista de Psicología del Deporte, 21(1), 195-200.

Godoy-Izquierdo, D., Lara, R., Vázquez, M.L., Araque, F. y Godoy, J.F. (2012). Correlates of Happiness Among Older Spanish Institutionalised and Non-Institutionalised Adults. Journal of Happiness Studies, 13(2).

Godoy-Izquierdo, D., Martínez, A. y Godoy, J.F. (2009). Balance afectivo en hombres y mujeres: Implicaciones de la edad y el sexo. Revista Internacional de Psicología Clínica y de la Salud, 2, 299-320.

Guillén, F. (1996). Calidad de vida y actividad física en la vejez: una perspectiva psicosocial. En M. Navarro, E. Brito, J.M. y García, J.A. Programas de Actividad Física y deportiva para las personas mayores, 179-198. Las Palmas de Gran Canaria: Exmo. Cabildo Insular de Gran Canaria.

Guillén, F. y Sánchez, M.C. (2010). La intervención del psicólogo/a del ejercicio y el deporte en la mejora de la salud y la calidad de vida en poblaciones especiales. Apuntes de Psicología, 28(2), 329-340.

Haring-Hidore, M., Stock, W.A., Okun, M.A. y Witter, R.A. (1985). Marital status and subjective well-being: a research synthesis. Journal of marriage and the family, 47(4), 947-958.

IBM Corp. Released 2012. IBM SPSS Statistics for Windows, Version 20.0. Armonk, NY: IBM Corp.

Jürgens, I. (2006). Práctica deportiva y percepción de calidad de vida. Revista Internacional de Medicina y Ciencias de la Actividad Física y el Deporte, 6(22), 62-74.

Kennedy, Q., Mather, M. y Carstensen, L.L. (2004). The role of motivation in the age-related positivity effect in autobiographical memory. Psychological Science, 15, 208-214.

Lacey, H.P., Smith, D.M. y Ubel, P.A. (2006). Hope I die before I get old: Mispredicting happiness across the adult lifespan. Journal of Happiness Studies, 7, 167-182.

Lang, F.R. y Heckhausen, J. (2001). Perceived control over development and subjective wellbeing: Differential benefits across adulthood. Journal of Personality and Social Psychology, 81, 509-523.

Lelkes, O. (2008). Happiness across the life cycle: Exploring age-specific preferences. European Centre for Social Welfare Policy and Research, Policy Brief, March (2), 1-16.

Liang, J., Lawrence, R.H. y Bollen, K.A. (1987). Race Differences in Factorial Structures of Two Measures of Subjective Well-being. The Journal of Gerontology, 42(4), 426-428.

Lucas, R.E. (2007). Long-term disability is associated with lasting changes in subjective wellbeing: Evidence from two nationally representative longitudinal studies. Journal of Personality and Social Psychology, 92, 717-730.

Márquez, D.X., Hu, L., Jerome, G.J. y Diener, E. (2005). Physical Activity Enhances Long-Term Quality of Life in Older Adults: efficacy, esteem and affective influences. Annals of Behavioral Medicine, 31(1), 99-103. 
Mckenzie, B. y Campbell, J. (1987). Race, socioeconomic status and subjective wellbeing of older Americans. The International Journal of Aging and Human Development, 25(1), 43-61.

McAuley, E., Blissmer, B., Katula, J., Duncan, T.E. y Milhako, S.L. (2000). Physical activity, selfesteem and self-efficacy relationships in older adults: a randomized controlled trial. Annals of Behavioral Medicine, 22, 131-139.

McAuley, E., Konopack, J.F., Motl, R.W., Morris, K.S., Doerksen, S.E. y Rosengren, K.R. (2006). Physical Activity and Quality of Life in Older Adults: Influence of Health Status and Self-Efficacy. Annals of behavioral Medicine, 31(1), 99-103.

McMahan, E.A. y Estes, D. (2012). Age-Related Differences in Lay Conceptions of Well-Being and Experienced Well-Being. Journal of Happiness Studies, 13, 79-101.

Meléndez, J.C., Tomás, J.M., Oliver, A. y Navarro, E. (2008). Psychological and physical dimensions explaining life satisfaction among the elderly: A structural model examination. Archives of Gerontology and Geriatrics, 48(3), 291-295.

Menec, V.H. (2003). The relation between everyday activities and successful aging: A 6-year longitudinal study. Journal of Gerontology. Series B, Psychological Sciences and Social Sciences, 58, S74-S82.

Mroczek, D.K. y Spiro, A. (2005). Change in life satisfaction during adulthood: Findings from the Veterans affairs normative aging study. Journal of Personality and Social Psychology, 88, 189-202.

Okun, M.A., Stock, W.A., Haring, M.J. y Witter, R.A. (1984). Health and Subjective Well-Being: A Meta-Analysis. The International Journal of Aging and Human Development, 19(2), 111-132.

Organización Mundial de la Salud. GPAQ: Global Physical Activity Questionnaire (versión 2.0 en $\begin{array}{lllll}\text { español). } & \text { Accedido } 20 & \text { de } & \text { septiembre }\end{array}$ http://www.who.int/chp/steps/instrument/es/index.html

Pawlowski, T., Downward, P. y Rasciute, S. (2011). Subjective well-being in European countries. On the age-specific impact of physical activity. European Review of Aging and Physical Activity, 8, 93-102.

Pinquart, M. (2001). Age differences in perceived positive affect, negative affect, and affect balance in middle and old age. Journal of Happiness Studies, 2, 375-405.

Pinquart, M. y Sorensen, S. (2000). Influences of socioeconomic status, social network, and competence on subjective well-being in later life: A meta-analysis. Psychology and Aging, 15(2), 187-224.

Pinquart, M. y Sorensen, S. (2001). Gender Differences in Self-Concept and Psychological WellBeing in Old Age. A Meta-Analysis. The Journals of Gerontology, series B, 56(4), 195213.

Rejeski, W.J., Brawley, L.R. y Shumaker, S.A. (1996). Physical activity and health-related quality of life. Exercise and Sports Sciences Reviews, 24, 71-108.

Salmon, J., Owen, N., Crawford, D., Bauman, A. y Sallis, J.F. (2003). Physical Activity and sedentary behavior: a population-based study of barriers, enjoyment and preference. Health Psychology, 22, 178-188.

Sánchez, P.A., Ureña, F. y Garcés de los Fayos, E.J. (2002). Repercusiones de un programa de actividad física gerontológica sobre la aptitud física, autoestima, depresión y afectividad. Cuadernos de Psicología del Deporte, 2(2), 57-73.

Suh, E., Diener, E. y Fujita, F. (1996). Events and subjective well-being: Only recent events matter. Journal of Personality and Social Psychology, 70, 1091-1102.

Suh, E., Diener, E., Oishi, S. y Triandis, H. (1998). The shifting basis of life satisfaction judgments across cultures: Emotions versus norms. Journal of Personality and Social Psychology, 74, 482-493. 
VanPraag, Frijters y Ferrer-i-Carbonell (2003). The anatomy of subjective well-being. Journal of Economic Behavior \& Organization, 51(1), 29-49.

Veenhomen, E. (2000). The four qualities of life. Ordering Concepts and Measures of the Good Life. Journal of Happiness Studies, 1(1), 1-34.

Waldinger, R.J. y Schulz, M.S. (2010). What's love got to do with it? Social functioning, perceived health, and daily happiness in married octogenarians. Psychology and Aging, $25,422-431$.

Recibido: 19 de mayo de 2013

Recepción Modificaciones: 24 de junio de 2013

Aceptado: 16 de septiembre de 2013 